Dagmar Dreyer

\title{
Zwischen Alter und Neuer Welt: Shakespeares Sturm
}

\section{Einleitung}

Der Sturm, so konstatiert David Scott Kastan, sei viel offensichtlicher ein Stück über europäische dynastische Belange als über europäische koloniale Aktivitäten (231). Über eine solche Bewertung gehen Forschungsmeinungen naturgemäß weit auseinander. Hier wird zunächst auf beide Aspekte eingegangen, zum einen auf die Störung und Wiederherstellung gesellschaftlicher Ordnung mit Bezug auf die Alte Welt. Zum anderen auf Shakespeares explizite Referenzen auf die Neue Welt, die im Zusammenhang mit kolonialen Eroberungs- und Entdeckungsreisen zu verstehen sind. In dieser Terminologie, das nur am Rande, umfasst die Alte Welt Europa, Afrika, Asien - also die Kontinente, die den Europäern vor der Entdekkung Amerikas bekannt waren (im Sturm selbst primär Europa) - , die Neue Welt Nord- und Südamerika.

Dann wird die Figurenkonstellation Prospero - Caliban im Detail betrachtet, da diese von besonderem Interesse für postkoloniale Lesarten und ,Um- 
Schreibungen' ist, die in ihr das Symbol schlechthin für das Verhältnis zwischen Unterdrücker und Unterdrücktem sehen. Zu diesem postkolonialen Kontext werde ich einen Überblick geben, dabei auch einen kurzen Ausflug in den theoretischen Bereich unternehmen. Abschließend wird das zu diesem Aspekt Skizzierte am Beispiel einer karibischen Shakespeare-Adaptation - Aimé Césaire, Ein Sturm (1969) - näher erläutert.

\section{Shakespeare, Der Sturm (1610/11)}

\subsection{Alte Welt: Macht und (Un-)Ordnung}

Die Macht-Verstrickungen der ,Alten Welt` erläutert Prospero seiner Tochter Miranda in der zweiten Szene des ersten Akts - im Anschluss an die eröffnende Sturmszene - und liefert somit auch den Zuschauern die für die Handlung wesentliche Vorgeschichte. Demnach wurde Prospero, der rechtmäßige Herzog von Mailand, zwölf Jahre zuvor von seinem machtgierigen Bruder Antonio gestürzt. Zu diesem Zweck hatte sich Antonio mit Alonso, dem König von Neapel, verbündet. Prospero und Miranda (damals ein dreijähriges Kind) wurden auf einem kaum noch funktionsfähigen Schiff in den vermeintlich sicheren Tod geschickt, doch sie strandeten auf der Insel, dem jetztigen Schauplatz, und überlebten. Der von Prospero eingangs heraufbeschworene Sturm bringt die an seinem Umsturz wesentlich Beteiligten, Antonio, Alonso, dessen Bruder Sebastian, außerdem seinen Sohn Ferdinand sowie einige Höflinge ebenfalls auf die Insel. Diese ,Notlandung' wiederum erfolgt auf ihrer Heimfahrt von Tunis; dort hatte kurz zuvor die Hochzeit von Alonsos Tochter Claribel mit dem König von Tunis stattgefunden. Somit lässt sich vorerst festhalten, dass die Schiffbrüchigen in diesem Zusammenhang keine kolonialen Intentionen hegen (vgl. Kastan 231).

Geographisch befindet sich die von Shakespeare interessanterweise als ,unbewohnt[]" bezeichnete Insel (vgl. Dramatis Personae) folglich im Mittelmeer, irgendwo zwischen Italien und Afrika. Die Herrschaftsbereiche, um die es in erster Linie geht, Mailand und Neapel, sind aber klar in der ,Alten Welt' verortet. Damit eng verbunden, werden Rebellion und Usurpation (durch Antonio) als wesentliche Themen eingeführt. ${ }^{58}$

58 Mailand und Neapel stehen darüber hinaus jedoch stellvertretend als Beispiele für autonome Herrschaftsbereiche, so dass eine allgemeine Lesart ebenfalls möglich ist und man von konkret politischen Assoziationen durchaus abstrahieren kann. In diesem Sinne heben Vaughan und Vaughan die dem Stück zugrunde liegenden menschlichen Beziehungen hervor, nämlich zwischen Vater und Tochter, König und Untergebenen sowie zwischen Herr und Diener, und die sich daraus ergebenden Wechselwirkungen von ,Freiheit und Einschränkung, Gehorsam und Rebellion, Autorität und Tyrannei‘ (Vaughan und Vaughan, Tempest 73-74). 
Im zweiten Akt fasst Antonio (der unrechtmäßige Herzog von Mailand) den Plan, seine politische Macht weiter auszubauen und seinen einstigen Verbündeten Alonso, dem Mailand nun untergeordet ist, zu ermorden. Dazu stiftet Antonio Alonsos Bruder Sebastian an, der sich an ihm ein Beispiel nehmen will:

Mein Freund,

Dein Fall zeigt mir den Weg: wie du zu Mailand, Komm' ich zu Napel.

$$
\text { (II, 1, 290-92) } 59
$$

Der Königsmord wird jedoch von Ariel, dem ,dienstbaren Geist' Prosperos, vereitelt, der die potentiellen Opfer rechtzeitig weckt. Auch hier, so sehen wir, geht es nicht die Eroberung von Neuland, sondern um eine Neuaufteilung italienischer Königreiche, mithin um europäische Machtinteressen.

In Akt III lässt Prospero ein Festessen für die bislang von seiner Anwesenheit nichts ahnenden ,Gäste ' auffahren, um sie dann von Ariel an ihre Verbrechen erinnern zu lassen. Während er damit zumindest bei Alonso Gewissensbisse auslöst, rüsten sich Antonio und Sebastian für ihren ,Staatsstreich'. Gonzalo kommentiert dies unter Bezugnahme auf die Schuld, die die Verräter durch ihre damalige Tat bereits auf sich geladen haben:

Sie alle drei verzweifeln; ihre große Schuld,

Wie Gift, das lang' nachher erst wirken soll,

Beginnt sie jetzt zu nagen. Ich ersuch euch,

Die ihr gelenker seid, folgt ihnen nach,

Und hindert sie an dem, wozu der Wahnsinn

Sie etwa treiben könnte. ${ }^{60}$

$$
\text { (III, 3, 105-110) }
$$

Seine Rede illustriert die destruktiven Auswirkungen einer so tiefgreifenden Störung der herrschenden Ordnung auf die Täter selbst und beendet den dritten Akt. Erst im fünften Akt sehen wir König und Gefolgsleute wieder, hier werden sie nun explizit als Gefangene Prosperos bezeichnet; er gibt sich ihnen schließlich zu erkennen, erinnert sie nochmals an das von ihnen begangene Unrecht, vergibt ihnen jedoch, obgleich nur Alonso echte Reue zeigt. Der in unserem Kontext

59 „Thy case, dear friend, / Shall be my precedent; as thou got'st Milan, / I'll come by Naples.“ (II, 1, 292-94) - Die englischen Zitate sind der von Virginia Mason Vaughan und Alden T. Vaughan herausgegebenen kritischen Arden-Edition entnommen, die deutschen Zitate der von L.L Schücking herausgegebenen Rowohlt-Ausgabe in der Übersetzung von Schlegel und Tieck. So erklären sich gelegentliche Abweichungen in den Versangaben. Bei Übereinstimmung erfolgt die Zitatangabe nach der deutschen Übersetzung.

60 „All three of them are desperate: their great guilt, / Like poison, given to work a great time after, / Now 'gins to bite the spirits. I do beseech you, / That are of suppler joints, follow them swiftly, / And hinder them from what this ecstasy / May now provoke them to." 
zentrale Aspekt ist die Wiederherstellung der Ordnung; Prospero wird als rechtmäßiger Machthaber re-installiert.

Aber auch auf einer weiteren Ebene wird die Versöhnung zwischen Mailand und Neapel besiegelt, nämlich durch eine Heirat der beiden Kinder und Erben, Miranda und Ferdinand, die sich bei ihrem ersten Zusammentreffen prompt ineinander verliebten.

Gehen wir kurz zurück zum ersten Akt, so sehen wir nicht nur Mirandas naives Staunen über den dritten Mann, dem sie - neben Prospero und Caliban - je in ihrem Leben begegnet ist, sondern auch Prosperos Strategie, die beiden einem Liebestest zu unterziehen:

Eins ist des anderen ganz: den schnellen Handel

Muß ich erschweren, daß nicht leichter Sieg

Den Preis verringere. ${ }^{61}$

$$
\text { (I, 2, 450-52) }
$$

Zu diesem Zweck betont er Ferdinand gegenüber seine Macht, behandelt ihn äußerst unfreundlich, bezichtigt ihn, ein Spion und Verräter zu sein und lässt ihn körperlich hart arbeiten. Doch schon am Anfang des vierten Aktes belohnt er Ferdinand für seine Mühen, indem er ihm Miranda wie einen Preis, überreicht‘:

$$
[\ldots] \mathrm{du}
$$

Hast die Probe wunderbar bestanden.

Hier vor des Himmels Angesicht bestät'ge

Ich dies mein reich Geschenk [...]

Als Gabe denn und selbsterworbnes Gut,

Würdig erkauft, nimm meine Tochter. ${ }^{62}$

$$
\text { (IV, 1, 6-8 und 13-14) }
$$

Man beachte das Vokabular, das diese ,Transaktion' beschreibt und Miranda gleichsam zum Objekt degradiert (vgl. Singh 214-15). Schlagen wir den Bogen wieder zur Versöhnungsszene im letzten Akt. Von Bedeutung ist die enge Verzahnung von privatem und politischem Kontext, der Liebeshandlung also und ihrer Funktion für eine dynastische Stabilisierung zwischen zwei vormals ungleichen Mächten (durch den Umsturz war ja Mailand gebenüber dem verbündeten Neapel tributpflichtig geworden). „Die Staats- und Gesellschaftsordnung, die am Ende von The Tempest wiederhergestellt wird,“ konstatiert Kullmann, „beruht auf dem Prinzip der Erbmonarchie und der auf dem Institut der Ehe basierenden Familie." (102)

61 „They are both in either's powers, but this swift business / I must uneasy make, lest too light winning / Make the prize light.“ (I, 2, 451-53)

$62,[\ldots]$ thou / Hast strangely stood the test. Here, afore heaven, / I ratify this my rich gift [...] / Then as my gift and thine own acquisition / Worthily purchased, take my daughter.“ 
Werfen wir einen Blick auf den historischen Hintergrund zur Entstehungszeit des Stücks (1610/11), so können wir eine weitere Parallele zur ,Alten Welt ${ }^{\star}$ und ihren politisch-dynastischen Belangen ziehen. Diese stellten in der Tat ein wichtiges Thema dar, beispielsweise im Hinblick auf den seit 1603 regierenden britischen King James I (Jakob I) und dessen Bestrebungen, „für seine Kinder politisch vorteilhafte Heiraten“ im Sinne diplomatischer Beziehungen zu europäischen Mächten zu initiieren (Kullmann 101). Seine Tochter Elizabeth verheiratete er mit dem ,ausländischen' protestantischen Prinzen Friedrich V. von der Pfalz. Diese Verbindung, deren Vorbereitungen bereits vor 1610 begannen, die aber erst 1613 stattfand und zu deren Feier Der Sturm aufgeführt wurde, bezeichnet Kastan als ,Politik mit anderen Mitteln - wie alle königlichen Hochzeiten' (233); politischnationale Interessen standen in der Regel klar im Vordergrund, so eben die (Wieder-)Herstellung friedlicher Beziehungen, auch und gerade über konfessionelle Gegensätze hinweg (vgl. Kastan 232-34; Kullmann 101; Vaughan und Vaughan, Tempest 37-38).

\subsection{Referenzen auf die Neue Welt}

Wenden wir uns nun den Referenzen hinsichtlich der ,Neuen Welt' zu. Welche Anhaltspunkte liefert uns Shakespeares Text? Der Schauplatz selbst, so hatten wir gesehen, ist nicht etwa im Atlantik, sondern im Mittelmeer angesiedelt und kann somit zunächst einmal nicht unmittelbar mit Amerika (einschließlich karibischer Inseln) als Neuer Welt identifiziert werden. Dennoch finden die Bermuda-Inseln im Nord-Atlantik Erwähnung; Ariel evoziert die „stürmischen Bermudas“ (I, 2, 229) als einen Ort, an dem er magische Zutaten beschaffte und illustriert auf diese Weise verschiedene zeitgenössische Konnotationen: mit heftigen, für Schiffe gefährlichen Stürmen, aber auch mit Zauberei und allerlei furchteinflößenden Dingen - in diesem Sinne galten die Bermudas übrigens als ,Teufelsinselnn ${ }^{63}$, und in gewisser Weise ist das durchaus auch für Prosperos bzw. Calibans Insel zutreffend.

Als Alonso, Antonio, Sebastian und Gonzalo einen Teil dieser Insel erkunden, u.a. um den Rest ihrer Mannschaft und insbesondere Ferdinand wiederzufinden Alonso befürchtet, sein Sohn sei ertrunken -, gibt ihnen das von Prospero inszenierte Bankett mit seiner „liebliche[n] Musik“ und seinen „seltsame[n] Gestalten“ (III, 3, 19) Anlass zur Verwunderung. Diese drückt sich in Bezugnahmen auf mythologische Wesen, teils Reiseberichten entnommen, aus. Nun, sagt Sebastian, könne er glauben, dass es auch Einhörner gebe und dass in Arabien Phoenixe existierten. Gonzalo erwähnt Erzählungen von Menschen, deren Kopf in ihrer

63 In der Annotation der Arden-Ausgabe heißt es dazu: William Strachey ,with a touch of hyperbole, called Bermuda 'the dangerous and dreaded ... Ilands', notorious for 'tempests, thunders, and other fearfull objects ... seene and heard about them', and known as the 'Devil's Ilands" (Vaughan und Vaughan, Tempest 165). 
Brust sitzt (III, 3, 46-47), und bezieht sich damit auf phantastisch-monströse Darstellungen von ,Wilden' und Menschenfressern, etwa in den Reiseschilderungen Mandevilles aus dem 14. Jahrhundert (vgl. Kermode xxxii).

Konfrontiert mit Caliban, weiß Trinculo nicht so recht, was er mit dem merkwürdigen, ,missgestalteten ${ }^{6}$ Wesen anfangen soll; er ordnet es zunächst irgendwo zwischen Fisch, Monster und Mensch ein und denkt auch gleich daran, wie er aus dessen Zurschaustellung in seiner Heimat Profit schlagen könnte, denn „(..) jedes fremde Tier macht dort seinen Mann: wenn sie keinen Deut geben wollen, einem lahmen Bettler zu helfen, so wenden sie zehn dran, einen toten Indianer zu sehen." (II, 2, 30-32) [,,[...] any strange beast there makes a man. When they will not give a doit to relieve a lame beggar, they will lay out ten to see a dead Indian." .

Seit 1576 wurden im Zuge der Nordamerika-Expeditionen des englischen Seefahrers Martin Frobisher (etwa auf der Suche nach der Nordwestpassage) immer wieder sogenannte Eingeborene (,Indianer' bzw. Inuit) nach England gebracht und dort gegen Geld ausgestellt (Vaughan und Vaughan, Tempest 208-09). Wieder gibt uns der Text also eher beiläufig einen Hinweis in Richtung kolonialer Unternehmungen der Zeit, doch wird auch klar, dass Trinculo Caliban nicht wirklich für einen ,Indianer', sondern für ein ,Monster ${ }^{6}$ hält. ${ }^{64}$

Nicht zuletzt stellt der Name,Caliban' selbst eine Anspielung auf die Neue Welt dar, kann er doch als Anagramm von ,cannibal' interpretiert werden, das etymologisch wiederum auf eine Form von ,Carib` zurückzuführen ist und einen ,wilden Bewohner der Neuen Welt' bezeichnet, der oftmals auch mit Kannibalismus assoziierte wurde (vgl. Kermode xxxviii; Vaughan und Vaughan, Tempest 31) ${ }^{65}$. Michel de Montaignes Essay „Of the Caniballes“ über Indianerstämme in Brasilien - in der englischen Übersetzung von 1603 - ist hier als eine Quelle zu nennen (Schabert 479-80), die Shakespeare allerdings in einem anderen Zusammenhang zitiert. So entwirft Gonzalo die Utopie einer im Einklang mit der Natur lebenden Insel-Gemeinschaft, die offenbar einen Gegenentwurf zur europäischen Zivilisation intendiert (vgl. II, 1, 148-169) und Montaigne entnommen ist. Gleichzeitig aber führt er seine Vision ad absurdum, indem er sich selbst als Herrscher in einem solchen Szenario imaginiert.

64 Ebensowenig definiert Calibans Beschreibung ihn eindeutig (vgl. Kastan 230). Prospero etwa bezeichnet ihn als „scheckig Wechselbalg“ einer „blau-äugigen Hexe“, „gezeugt vom Teufel selbst“ (I, 2, 282; 269; 319-20). Während ältere Interpretationen dies als Bezug zu Vorstellungen der Alten Welt auffassen (vgl. Kermode xl), fokussieren jüngere Ansätze stärker die algerische Herkunft seiner Mutter Sycorax, die Tatsache, dass sie bereits schwanger war, bevor sie auf die Insel verbannt wurde, und den sich daraus ergebenden ,Ursprung' Calibans in Afrika (vgl. Vaughan und Vaughan, Tempest 48).

65 Die von Vaughan und Vaughan herausgegebene Arden-Edition verweist aber auch auf alternative Etymologien, u.a. auf ,Calibia' als afrikanische topographische Bezeichnung nahe der Mittelmeerküste (vgl. 32). 
Als letztes Beispiel für eine direkte Textreferenz zur Neuen Welt mag Calibans Nennung von Setebos dienen, einer Gottheit der patagonischen ,Indianer', die im christlichen Verständnis dämonisiert wurde (vgl. Singh 216).

Diese Beispiele und ihre Erläuterungen sollten verdeutlichen, dass die relativ wenigen unmittelbaren Bezugnahmen auf die Neue Welt meist nur kurz ausfallen, überwiegend in Form von Namen (Bermudas, Setebos, Caliban-Kannibale). Sie stehen jedoch im Kontext eines breiten Diskurses europäisch-kolonialer Entdekkungs- und Eroberungsreisen, mit dem Shakespeare durchaus vertraut war. Folgende Quellen werden hier in der Regel aufgeführt: In Robert Edens History of Travaile (1577) findet sich die englische Übersetzung von Antonio Pigafettas Bericht über Magellans Südamerika-Umseglung, 1519-22 (vgl. Vaughan und Vaughan, Tempest 40; Kermode xxxii). Sylvester Jourdains Discovery of the Barmudas (1610) berichtet von der zufälligen Entdeckung der Bermudas 1609, als englische Schiffe auf der Fahrt in die Kolonie Virginia in einen Sturm gerieten und ein Schiff auf den Inseln strandete; ebenso William Strachey, True Repertory of the Wrack (im handschriftlichen Original von 1610; veröffentlicht 1625, vgl. Kermode xxvivii).

Was sich ebenfalls abzeichnet, ist die Tendenz, europäische Identität mit teils monströs-grotesken Bildern von der Andersartigkeit fremder Völker zu konstrastieren (vgl. Singh 215; auf einen sehr ähnlichen Aspekt wird später noch eingegangen).

\subsection{Prospero und Caliban}

Insbesondere die Konstellation Prospero - Caliban hat ein weitreichendes Echo in der Rezeption von Shakespeares Sturm gefunden, scheint doch hier das Verhältnis zwischen Kolonisator und Kolonisiertem prototypisch gefasst. Betrachten wir also Shakespeares Figurengestaltung und dazu einführend zwei Bilder aus dem 18. Jahrhundert (Füssli der Boydell Gallery entnommen), die Ähnlichkeiten in der Darstellung Prosperos und Calibans aufweisen (vgl. Abb. 1 und 2).

So wird Prospero als fast gottgleicher weiser alter Mann in Szene gesetzt, dessen Dominanz insbesondere bei Füssli zum Ausdruck zu kommen scheint; bei Hogarth und Füssli sieht man sehr schön den tierähnlichen Caliban respektive die Zwischenform zwischen Mensch und Tier. Hogarth betont seine ,Missgestaltung'; interessant sind in diesem Zusammenhang die Flossen-Füße, die wohl auf eine Verwandtschaft mit amphibischen Lebewesen hindeuten könnten. ${ }^{66}$ Eine ausführliche Bildinterpretation, die darlegt, dass der erste Eindruck hinsichtlich Prosperos Rolle der Modifizierung bedarf, findet sich im Ausstellungskatalog zur Boydell Gallery (speziell im Artikel von Grant F. Scott).

66 Im 19. Jahrhundert wurde Caliban symbolisch als Bindeglied in der evolutionären Entwicklung des Menschen aus ,einer Art Wassertier' gesehen, so in Daniel Wisons Caliban: The Missing Link (1873), vgl. Vaughan und Vaughan, Tempest 91. 


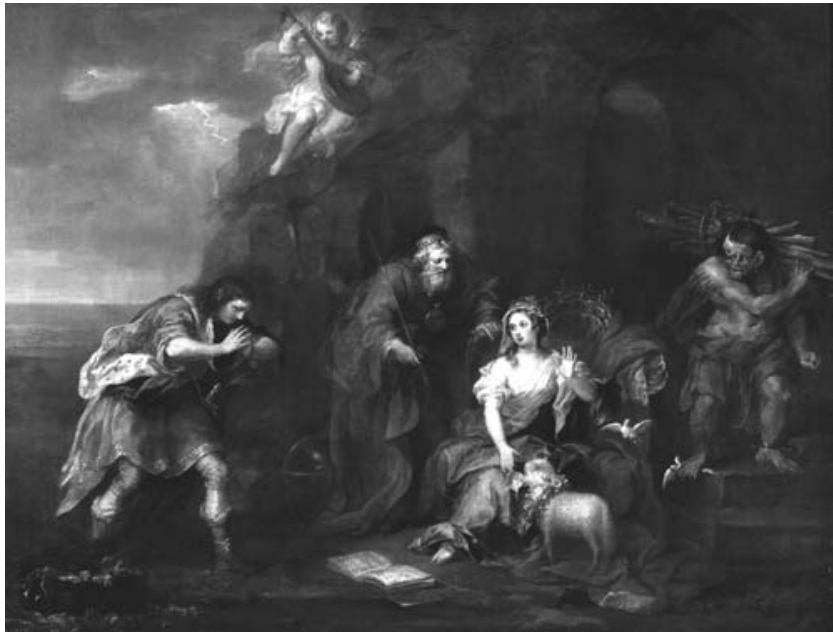

Abb. 1a. W. Hogarth, A Scene from The Tempest

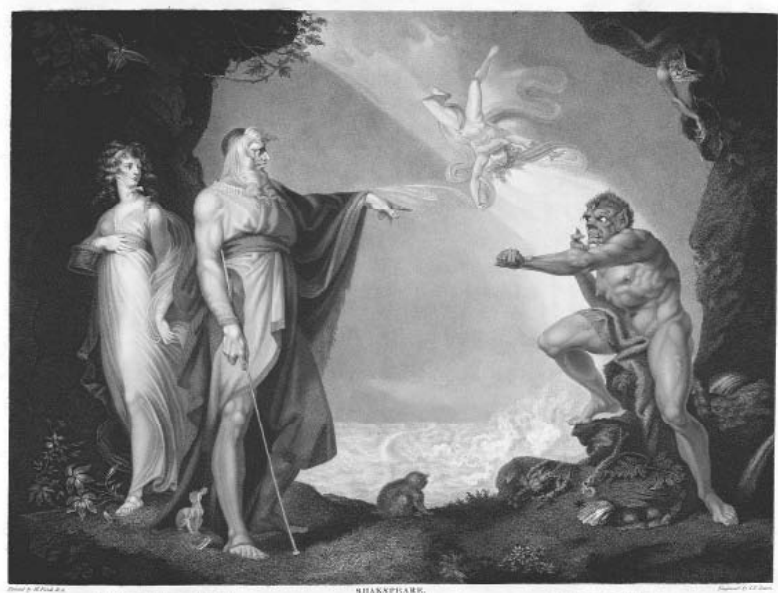

moment

Abb. 2a. J. H. Füssli, The Tempest (Act I, Scene 2)

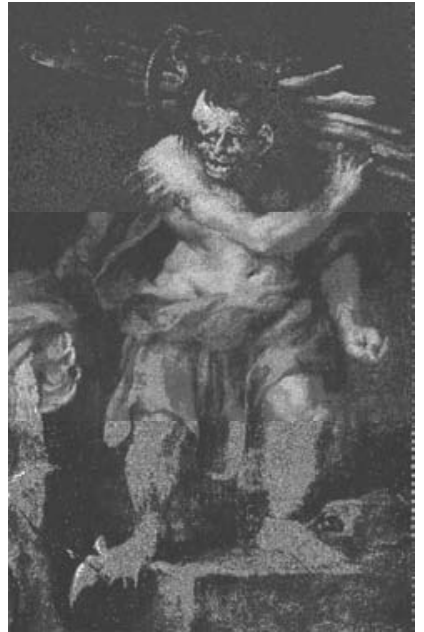

Abb. 1b: Detail: Caliban

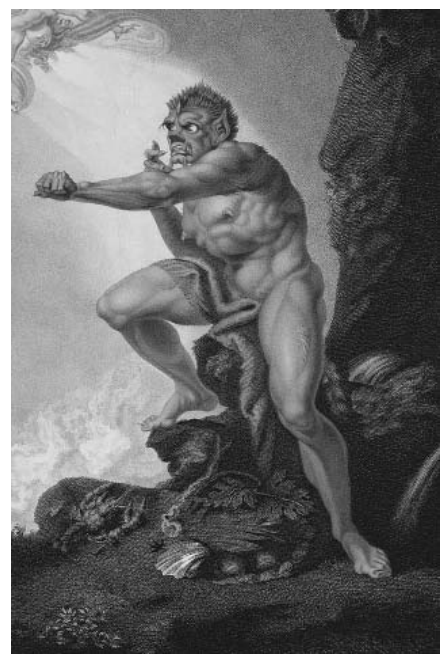

Abb. 2b: Detail: Caliban 
Bevor Caliban bei Shakespeare die Szene betritt, findet ein Austausch zwischen Prospero und dem als Luftgeist konzipierten Ariel statt, der die Themen um Dienen, Unterwerfung und Freiheit bereits anschneidet. Ariel, von Prospero beordert, grüßt ihn mit „Heil, großer Meister!“ (I, 2, 189), erstattet detailliert Bericht über die sorgfältige Ausführung seines Auftrags, die Schiffsmannschaft durch den Sturm auf der Insel stranden zu lassen und zu zerstreuen und erhält dafür ausgiebiges Lob. Als Prospero aber andeutet, es gebe noch weitere Aufgaben zu erledigen, erinnert Ariel ihn an sein Versprechen, ihn im Gegenzug für seine Mühen freizulassen. Schlagartig ändert sich das Verhalten seines Gegenübers. Prospero sieht offenbar seine Autorität in Frage gestellt und addressiert Ariel nunmehr nicht nur als „boshaftes Ding“ und „mein[en] Sklav[en]“, sondern erinnert ihn daran, wie er ihn einst aus der Gefangenschaft der Hexe Sycorax - Calibans Mutter - befreite und droht, ihn bei weiterem ,Murren' wiederum zwölf Jahre in einen gespaltenen Baum einzukeilen (vgl. I, 2, 257-97). Schon ein so geringfügiger Anlass wie Ariels minimales Aufbegehren - eigentlich eher eine Nachfrage - bringt Prospero also in Rage. Am Ende bedankt sich Ariel noch einmal für seine Jahre zurückliegende Befreiung, entschuldigt sich für seinen ,Ungehorsam' und preist Prosperos Güte, als der ihm nun zusagt, ihn in zwei Tagen endgültig freilassen zu wollen. Dabei apostrophiert er ihn nicht weniger als dreimal als „master“ (Meister bzw. Herr), wodurch er seine Unterordnung betont. Dies also bildet den Auftakt zur Konfrontation von Prospero, Caliban und der ebenfalls anwesenden Miranda (die auch in den Bildern von Hogarth und Füssli in einer Dreierkonstellation zu sehen ist).

Prosperos Haltung Caliban gegenüber ist von vornherein deutlich herrischer und abwertend, Fragen werden weitgehend durch Imperative ersetzt, und die Bezeichnung „Sklave“ fällt fünfmal, etwa in der Anrede: „What ho, slave! Caliban, / Thou earth, thou: speak!“ (I, 2, 314-15) [„He, Sklave! Caliban! Du Erdkloß, sprich!“ (313-14)]. Oder: „Thou poisonous slave, got by the devil himself / Upon the wicked dam; come forth!“ (I, 2, 320-21) [„,Du gift'ger Sklav, gezeugt vom Teufel selbst / Mit deiner bösen Mutter! komm heraus!“ (319-20)]

Beleidigungen und Flüche bestimmen den Beginn des Dialogs; zudem droht Prospero Caliban massiv mit körperlichen Schmerzen (Krämpfen, Stichen, Bissen) als Sanktionierungsmaßnahme und generiert sich auf diese Weise in der Tat recht drastisch als Unterdrücker. Es folgt Calibans berühmte Rede, in der er sich nach dem Tod seiner Mutter Sycorax als rechtmäßiger Besitzer der Insel präsentiert, der von Prospero erst ausgenutzt, dann unterworfen wurde:

\section{Dieses Eiland}

Ist mein, von meiner Mutter Sycorax,

Das du mir wegnimmst. Wie du erstlich kamst,

Da streicheltest du mich und hieltst auf mich

[...] da liebt' ich dich,

Und wies dir jede Eigenschaft der Insel 
$[\ldots]$

Fluch, daß ich's tat, mir! Alle Zauberei

Der Sycorax, Molch, Schröter, Fledermaus, befall' euch!

Denn ich bin, was ihr habt an Untertanen,

Mein eigner König sonst; und stallt mich hier

In diesen harten Fels, derweil ihr mir

Den Rest des Eilands wehrt. ${ }^{67}$

$$
\text { (I, 2, 330-344) }
$$

Prospero aber hält dagegen, er habe Caliban mit Güte behandelt, bis der sich an Miranda vergreifen wollte (um die Insel mit kleinen Calibans zu bevölkern); er verstünde eben nur Schläge, nicht Milde. Hier greift nun Miranda selbst ein und spricht Caliban jede „Spur des Guten“ (I, 2, 352) ab; aus Mitleid habe sie ihn ihre Sprache lehren wollen, doch aufgrund seiner niederen, tierischen Natur sei das ein vergebliches Unterfangen gewesen. Caliban kontert, der Nutzen dieser Sprache sei für ihn lediglich, dass er fluchen könne. Am Ende dieser Auseinandersetzung aber muss er sich fügen. Interessant ist dabei seine abschließende Bemerkung, er müsse gehorchen, da Prosperos magische Macht die bereits erwähnte Gottheit Setebos kontrollieren, bezwingen und zum „Vasallen“ (I, 2, 374) machen könne. Somit zieht er indirekt eine Parallele zu seinem eigenen Schicksal als nunmehr Abhängiger. Der thematische Aspekt des In-Besitznehmens und Beherrschens durch Prospero tritt hier klar zutage. Dazu gehört übrigens auch, dass Prospero keinerlei Parallelen zwischen seiner eigenen und Calibans Situation zieht; er selbst mag von seinem Bruder ,entthront ${ }^{6}$ worden sein, seinen Herrschaftsanspruch über die Insel gegenüber dem einheimischen Bewohner sieht er jedoch als Selbstverständlichkeit (vgl. Kastan 229).

Und obwohl sich Caliban von seinem Unterdrücker befreien will, geht es ihm nicht etwa um Freiheit an sich (trotz seines Schlachtrufes am Ende des zweiten Akts, „Freedom, high-day“ - „Heisa, heisa, Freiheit“), vielmehr wählt er neue Verbündete, denen er sich freiwillig unterwirft. Zu diesem Zweck bietet er seine Dienste ausgerechnet dem trinkfreudigen Butler Stephano sowie dem Narren Trinculo an (die ihn prompt betrunken machen), er umschmeichelt sie und präsentiert sich extrem unterwürfig: „Ich zeig“ dir jeden fruchtbaren Fleck der Insel, Und will den Fuß dir küssen; bitte, sei mein Gott!"“ (II, 2, 153-54) ${ }^{68}$.

67 „This island's mine by Sycorax, my mother, / Which thou tak'st from me. When thou cam'st first / Thou strok'st me and made much of me / [...] And then I loved thee / And showed thee all the qualities o'th isle / [...] / Cursed be I that did so! All the charms / Of Sycorax toads, beetles, bats - light on you, / For I am all the subjects that you have, / Which first was mine own king; and here you sty me / In this hard rock, whiles you do keep from me / The rest o'th' island.“ (I, 2, 332-45).

68 „'Ill show thee every fertile inch o'th' island, / And I will kiss your foot. I prithee, be my god.“ (II, 2, 145-46) 
Im Gegenzug sollen sie Prospero für ihn töten, wobei Caliban in seinen Instruktionen eine bemerkenswert gewalttätige Detailliertheit zeigt. ${ }^{69}$ Diese Verschwörung entfaltet sich parallel zu den Plänen Antonios und Sebastians, auch sie wird jedoch von Ariel belauscht und abgewendet, indem er Prospero warnt. Dessen Zorn darüber entlädt sich plötzlich mitten in dem von ihm inszenierten allegorischen Spiel zur Verlobung von Ferdinand und Miranda, das er prompt abbricht. Seine Charakterisierung von Caliban bringt dabei den Gegensatz zwischen negativ verstandener, deformierter, wilder Natur und Erziehung, letztlich Zivilisation (exemplifiziert auch in Prosperos Büchern) auf den Punkt:

Ein Teufel, ein geborner Teufel ist's,

An dessen Art Erziehung nimmer haftet,

An dem die Mühe, die ich menschlich nahm,

Ganz, ganz verloren ist, durchaus verloren. ${ }^{70}$

$$
\text { (IV, 1, 188-191) }
$$

In diesem Sinne scheinen die beiden Figuren zwei Extrempole zu repräsentieren, doch werden allzu einfache Grenzziehungen im Stück unterlaufen, wodurch die Charakterzeichnung an Ambivalenz gewinnt. Caliban wird eben nicht nur als wild, missgestaltet und fluchend beschrieben, sondern auch als empfänglich für Schönheit und Musik. In gewisser Weise ist er sogar Miranda in seiner Naivität und seinem Staunen über die gestrandeten ,Eindringlinge ähnlich. ${ }^{71}$ Auch sein Wunsch, mit ihr, der einzigen Frau, die er außer seiner Mutter kennengelernt hat und von der er sehr angetan ist, Nachkommen zu zeugen, muss nicht notwendigerweise Bösartigkeit und Verdorbenheit entspringen (vgl. Singh 214-215). Bösartigkeit und Verdorbenheit, Korruption durch ungezügelte Machtgier findet sich vor allem in Antonio, der derselben Welt entstammt wie Prospero (vgl. Kermode lii-liii). Er hat sich unrechtmäßig an die Macht gebracht und plant, sie durch einen Mord noch auszubauen, Caliban dagegen will sich von einem Tyrannen befreien, dessen Herrschaft über die Insel er als unrechtmäßig empfindet. Dass er dafür bereit ist, die erstbesten Dahergelaufenen als neue ,Herren' zu betrachten und zu vergöttern, lässt ihn als ,Sklave von Natur aus' erscheinen. ${ }^{72}$ Am Ende aber zeigt Caliban Ein-

69 „[...] There thou mayst brain him, / Having first seized his books, or with a log / Batter his skull, or paunch him with a stake, / Or cut his wezand with thy knife.“ (III, 2, 88-91) [,,...) da kannst ihn würgen, / Hast du erst seine Bücher; mit 'nem Klotz / Den Schädel ihm zerschlagen, oder ihn / Mit einem Pfahl ausweiden, oder auch / Mit deinem Messer ihm die Kehl' abschneiden.“ (III, 2, 95-100)]

70 „A devil, a born devil, on whose nature / Nurture can never stick; on whom my pains / Humanely taken - all, all lost, quite lost!“ (IV, 1, 188-190)

71 Generell werden jedoch die Unterschiede hervorgehoben, so beispielsweise von Schabert: „Mirandas naive Unschuld und Güte kontrastiert mit Calibans unversöhnlicher Wut, Ferdinands keusche Liebe mit Calibans ungezügelter Sexualität.“ (482)

72 Dies könnte jedoch auch die Folge der jahrelangen Unterdrückung durch Prospero sein, ist Caliban doch anfangs durchaus Herr seiner Insel. Auf diesen Aspekt werde ich im Zusammen- 
sicht und bittet Prospero - anders als Antonio und Sebastian - um Verzeihung; er wird auf der Insel zurückbleiben. Mirandas berühmter Ruf des Erstaunens beim Anblick der versammelten ,Mannschaft',

O Wunder!

Was gibt's für herrliche Geschöpfe hier!

Wie schön der Mensch ist! Wackre neue Welt,

Die solche Bürger trägt! ${ }^{73}$ (V, 1, 181-184)

entbehrt nicht einer gewissen Ironie, bedenkt man, dass zumindest drei der Anwesenden sich der Rebellion und Usurpation schuldig gemacht haben, zwei davon skrupellos noch ein weiteres schwerwiegendes Verbrechen begehen wollten und keinerlei Reue zeigen.

Schließlich haben wir gesehen, dass Prospero - entgegen einer langen Darstellungstradition als wohlwollender weiser Mann (vgl. Vaughan und Vaughan 24) keinesfalls nur positiv gezeichnet ist. ${ }^{74}$ Sein Umgang mit Caliban mag der Enttäuschung über das Versagen seiner Erziehungs- und Zivilisierungsversuche geschuldet sein, doch präsentiert er sich hier in der Tat tyrannisch. Prosperos magische Künste werden zwar mit der schwarzen Magie der Hexe Sycorax positiv kontrastiert, haben jedoch auch dazu geführt, dass er über seine Bücher die Herrscherpflichten vernachlässigte und seinen Sturz somit erleichterte; überdies enthalten sie durchaus dunklere Aspekte (vgl. Vaughan und Vaughan, Tempest 25; Greenblatt 231). So schwört er der ,Zauberei' im letzten Akt ab. Wie sich zeigt, hat die Kontrolle, die Prospero während der Dramenhandlung über seine Untergebenen, Feinde, sogar seine Tochter und Ferdinand ausübt - häufig wird er selbst als „Regisseur" gesehen (Schabert 480) -, ihre problematischen Seiten.

\section{Postkolonialer Kontext}

Verlassen wir nun das frühe 17. Jahrhundert und machen einen gewaltigen zeitlichen Sprung zu den sogenannten postkolonialen Literaturen, denjenigen

hang mit dem Konzept des kolonialen Diskurses und der Besprechung von Césaires Sturm eingehen.

73 „O wonder! / How many goodly creatures are there here! / How beauteous mankind is! O brave new world / That has such people in't." (V, 1, 181-184)

74 Vaughan und Vaughan skizzieren den Wandel im Verständnis der Prospero-Figur vom 18. bis zum 20. Jahrhundert und betonen Prosperos manipulativen Charakter (Tempest, 24). Vgl. auch Kastan zu Prosperos magischen Künsten, die nicht länger im Dienste, wohlwollender Zivilisation' gesehen werden, sondern - im postkolonialen Kontext - als Machtinstrumente eines Kolonisatoren: „In our anxious postcolonial moment, the power of Prospero's art, once confidently viewed as benevolently civilising, has become the coloniser's technology of domination and control.“ (227-28) Dagegen betonen ältere Interpretationen den Gegensatz zwischen der von Prospero verkörperten Magie bzw. Kunst als der Welt der Zivilisation zugehörig und Calibans deformierter und korrumpierter Natur, vgl. etwa Kermode xxxviii-xliii. 
englischsprachigen Literaturen also, die in den Kolonien des Britischen Empires vor und nach ihrer Unabhängigkeit entstanden sind bzw. natürlich noch immer entstehen, etwa in Indien respektive Südasien, Afrika oder in den ehemaligen ,weißen Siedlerkolonien“ wie Kanada, Australien und Neuseeland. Die Begriffe der postkolonialen, ,Neuen Englischsprachigen“ oder auch ,Anglophonen Literaturen“ werden - mit leicht variierenden Schwerpunkten - mehr oder minder synonym verwendet. Mag der Terminus ,post-kolonial' eine Nachzeitlichkeit in Bezug auf Kolonialismus suggerieren, so wird er doch in der Regel in einem weiteren Sinne verwendet, insbesondere in einem der zentralen Texte über postkoloniale Theorie und Literaturen, The Empire Writes Back (etwa Das Empire schreibt zurück) von 1989. Dort heißt es:

Wir benutzen den Begriff ,post-kolonial‘ (...), um damit alle vom imperialen Prozess beeinflusste Kultur vom Moment der Kolonisierung an bis zum heutigen Tag zu bezeichnen. Und zwar (sehr frei übersetzt) weil es noch immer Auswirkungen des Kolonialismus respektive kolonial motivierte Aktivitäten gibt. (Ü: DD) 75

Das Verhältnis zwischen Prospero und Caliban ist insbesondere in Zeiten der Entkolonialisierungsbestrebungen seit der Mitte des 20. Jahrhunderts immer wieder kritisch in den Blick genommen worden, sah man in ihm doch die ,zentrale ,koloniale“ Metapher" (Zabus, „Calibanic Tempest“ 35) überhaupt für die Beziehung zwischen Kolonisator und Kolonisiertem (im Englischen etwas einfacher ,coloniser and colonised ${ }^{\top}{ }^{76}$

Die erste kritische Studie zur Kolonialisierung, die sich Prosperos und Calibans als Prototypen bediente, wurde 1950 vom französischen Psychologen Octave Mannoni unter dem Titel La psychologie de la colonisation (Die Psychologie der Kolonialisierung) veröffentlicht; sie entstand unter Bezugnahme auf eine gescheiterte Rebellion in Madagaskar (damals französische Kolonie) in den Jahren 1947-48. Mannoni stellte die These auf, Caliban - als Repräsentant des kolonialisierten Subjekts leide an einem tief verwurzelten Abhängigkeitskomplex und benötige daher die

75 We use the term 'post-colonial' $[\ldots]$ to cover all the culture affected by the imperial process from the moment of colonization to the present day. This is because there is a continuity of preoccupations throughout the historical process initiated by European imperial aggression. (Ashcroft et. al. 2)

76 Vaughan und Vaughan erläutern zwei diametral entgegengesetzte Sichtweisen Calibans in lateinamerikanischen und afrikanischen Adaptionen, namentlich als Symbol für die Schattenseiten herrschender imperialistischer Staaten oder, insbesondere in den letzten Jahrzehnten, als Symbol für den unterdrückten Einheimischen: „Authors who invoke The Tempest in Latin America or African contexts have differed drastically over whom or what Caliban symbolizes. Diametrical opposites are proposed: Caliban as examplar of imperialist oppressors (the prevalent view in the late nineteenth and early twentieth centuries) or Caliban as emblem of oppressed natives (prevalent in recent decades). Advocates of the first approach found Shakespeare's monster a handy image for everything gross and vicious in a domineering nation or social class - Yankee imperialism, for example, or European racism. [...]“ (Vaughan und Vaughan, Caliban 145) 
Autorität eines kolonialen Beherrschers. Die Implikation, Caliban sei ein ,williger Partner' im Prozess seiner eigenen Unterwerfung (Nixon, 564-65) - nun aber nicht länger nur auf Shakespeares Drama, sondern verallgemeinernd auf die Mentalität kolonialiserter Völker bezogen - stieß (wenig überraschend) auf heftige Kritik. Erste Reaktionen kamen vom Schriftsteller und Politiker Aimé Césaire sowie dem Psychologen Frantz Fanon, beide aus Martinique (einer Insel der Kleinen Antillen in der Karibik). Beide kritisierten Mannoni für seine Reduktion eines komplexen Prozesses auf zwei psychologische Typen mit komplementären Veranlagungen: d.h. herrschen wollen und beherrscht werden wollen (vgl. Nixon 56265). Zugleich interpretierte Mannoni Caliban erstmals als Schwarzen bzw. Afrikaner (Vaughan und Vaughan, Caliban 159). Césaire publizierte seine Antwort auf Mannoni im selben Jahr, 1950, in Discours sur la colonialisme (Diskurs über den Kolonialismus) (Nixon 564). Darin argumentiert er unter anderem, dass Kolonialismus letztlich den Kolonisator ,entzivilisiere', da seine Machtinstrumente auf brutaler physischer, aber auch psychischer Gewalt beruhten und ihn somit auf die Stufe der Barbarei stellten; Europa degradiere sich damit selbst (vgl. Kelley xi). Fanon geht in Peau noire, masques blancs (Scbwarze Haut, weiße Masken) von 1952 einen Schritt weiter, wenn er feststellt: ,[... [i is the racist who creates his inferior“", es sei also der Rassist, der das Stereotyp vom Unterlegenen, Minderwertigen konstruiere (Zabus, „Calibanic Tempest“ 37).

Das karibische und afrikanische Interesse an Shakespeares Sturm dominierte zu einer Zeit (seit den späten 1950ern), als eine weitangelegte nationale Befreiung als möglich und unmittelbar bevorstehend gesehen wurde. Ein besonderes Augenmerk galt dabei der Figur des Caliban, dessen Rolle radikal uminterpretiert wurde (vgl. Nixon 566). In diesem Rahmen war einer der ersten karibischen Schriftsteller George Lamming. In Pleasures of Exile (Die Freuden des Exils) von 1960, einem Text, der politische, textkritische und autobiographische Elemente mischt, benutzt Lamming Shakespeare, um über die Geschichte der Karibik, über Auswirkungen und Konsequenzen des Kolonialismus zu schreiben und sich zugleich der Aufmerksamkeit eines westlichen Lesepublikums zu versichern (vgl. Nixon 566-69). Im Kapitel „A Monster, a Child, a Slave“ („Ein Monster, ein Kind, ein Sklave“) unterzieht er die ersten beiden Szenen im ersten Akt des Dramas einer Lektüre, die beispielsweise die physische Erfahrung des Sturms und des resultierenden Schiffbruchs mit der Erfahrung afrikanischer Sklaven auf ihrem qualvollen Transport zu den karibischen Kolonien parallel setzt. Seine Interpretation der Shakespearschen Figuren identifiziert Prospero als Imperialisten und Sadisten, Caliban u.a. als Opfer, aber auch proto-nationalistischen Führer. Überdies analysiert Lamming Prosperos vermeintliches, Geschenk der Sprache an Caliban, das für diesen jedoch eher ein Gefängnis darstellt, ein Paradigma, innerhalb dessen er immer Properos Sklave bleiben wird. Dies ist lediglich eine grobe Skizzierung der von Lamming thematisierten Sachverhalte - auf einen weiteren Text aus dem 
Zeitraum wird aber gleich noch ausführlicher eingegangen, und zwar auf die dramatische Adaptation des bereits erwähnten Aimé Césaire. ${ }^{77}$

Zuvor ist aber ein kurzer Ausflug in die Gefilde postkolonialer Theorien notwendig; und zwar geht es um folgende zwei Aspekte: zum einen um eine Theorie kolonialen Diskurses (Colonial Discourse), zum anderen um das Konzept des Writing Back (Zurück- oder Gegenschreiben); beide sind relevant für Césaires Stück.

Theorien kolonialer Diskurse erforschen, grob gesagt, wie Repräsentationen und Wahrnehmungsweisen gezielt als Instrumente kolonialer Macht benutzt und eingesetzt werden, um kolonialisierte Völker in einer untergeordneten Position zu halten (McLeod, 17); dabei geht es immer wieder um den psychologischen Prozess der Internalisierung. John McLeod, der eine sehr lesenswerte Einführung zum Postkolonialismus geschrieben hat, zitiert zur Veranschaulichung den trinidadischen Schriftsteller Sam Selvon mit folgender Begebenheit aus dessen Kindheit. Selvon erinnert sich an einen einheimischen Fischer aus Trinidad, Sammy, der teilweise gelähmt war und von Kindern oft verspottet wurde. Eines Tages brachte Sammy einen Weißen mit, der ihm assistierte. Selvon beschreibt sein Unverständnis und seinen Ärger darüber, dass ein karibischer Fischer einen weißen Mann als Helfer engagiert hatte - das schien ihm grundfalsch, denn der Weiße sollte doch wohl der ,Herr' sein, nicht Sammy. Selvon empfand beim Anblick diese weißen Mannes eine Sympathie und Bestürzung, die er noch nie für den gelähmten Sammy empfunden hatte. Mit dieser Anekdote will Selvon illustrieren, wie er als Kind gelernt hat, nicht-weiße Menschen als ,minderwertig' und untergeordnet zu betrachten, also eine bestimmte Weltsicht respektive Ordnung der Dinge zu verinnerlichen, und dies wiederum zeigt uns sehr gut, wie Kolonialismus besonders effektiv funktionieren konnte (vgl. McLeod 17).

In diesem Zusammenhang ist natürlich Sprache eines der zentralen Instrumente, transportiert sie doch Weltsichten, Werte und Normen; mit Hilfe von Sprache ordnen und erklären wir uns die Welt. Der kenianische Schriftsteller Ngugi wa Thiong'o hat das folgendermaßen auf den Punkt gebracht:

Sprache vermittelt Kultur, und Kultur vermittelt, insbesondere durch mündliche Überlieferung und Literatur, den gesamten Bestand an Werten, anhand derer wir uns selbst und unseren Platz in der Welt definieren. (Ü: DD) $)^{78}$

77 Weitere Beispiele für karibische und afrikanische Adaptionen sind Edward Brathwaites Gedichtband Islands (1969), George Lammings Roman Water with Berries (1971) und David Wallaces Drama Do You Love Me Master? (1971/77). - Englischsprachige kanadische rewritings fokussieren das Verhältnis zwischen Prospero und Miranda; vermutlich in Analogie zu Kanadas Status als weißer Siedlerkolonie sind hier nicht Sklaven von primärem Interesse, sondern die patriarchal dominierte Eltern-Kind-Beziehung (vgl. z.B. Zabus, „Calibanic Tempest“ 42-45).

78 „Languge carries culture, and culture carries, particularly through orature and literature, the entire body of values by which we come to perceive ourselves and our place in the world." (wa Thiong'o 441) 
Der Titel Decolonising the Mind (etwa Entkolonialiserung des Bewusstseins) von 1986 war Programm; wa Thiong'o war - zumindest zeitweilig - dazu übergegangen, nicht mehr auf Englisch, sondern in seiner Muttersprache Gikuyu zu schreiben.

Frantz Fanon, von dem bereits die Rede war, analysiert in Schwarze Haut, Weiße Masken die zerstörerischen Auswirkungen der Internalisierung auf das Selbstbild unterdrückter Menschen. Ihm zufolge, hat der koloniale Beherrscher die Definitionsmacht, er konstruiert die Identität des Kolonisierten; der wiederum übernimmt und verinnerlicht diese Identität. Dies kann soweit gehen, dass zu einer Art Bewusstseinsspaltung kommt, in der sich das kolonialisierte Subjekt selbst als Objekt betrachtet, sich selbst mit den Augen des Kolonisators als ,das Andere' sieht, als inferior. Fanon führt als Beispiel den konstruierten Gegensatz zwischen dem ,weißen Kolonisator ${ }^{6}$ und dem ,Negro \%,Neger ${ }^{6}$ an. Ersterer wird als rational, zivilisiert und intelligent gesehen, letzterer repräsentiert dazu in Negation ,das Anderé: Irrationalismus, barbarische Wildheit, Dummheit. Aber, und das ist das von Fanon konstatierte ultimative Dilemma, der derart diskriminierte Schwarze kann noch so sehr weiße Erziehung, weiße Werte und die Sprache des Kolonialmacht übernehmen wollen - quasi eine weiße Maske aufsetzen -, er wird nie als gleichwertig akzeptiert werden (vgl. McLeod 19-21).79 Soweit zunächst zum kolonialen Diskurs.

Was hat es nun mit Writing Back auf sich? Stellen wir uns eine weitere Dichotomie vor, nämlich zwischen Zentrum und Peripherie. Vom Britischen Empire aus gedacht, stellt Großbritannien das Zentrum dar, seine (Ex-)Kolonien (z.B. Indien, Afrika, Kanada) liegen dagegen an den Rändern. Diese räumliche Dimension kann nun aber wieder auf eine ganz andere Ebene verlagert werden, so dass englische Kultur (etwa der klassische Kanon englischer Literatur), englische Werte und natürlich die englische Sprache ebenfalls das ,Zentrum' bestimmen und der Peripherie gegenüber als, überlegen' gewertet werden; sie repräsentieren den geltenden Maßstab (vgl. Ashcroft et. al. 3). In diesem Sinne bedeutet ,Zurück- bzw. Umschreiben' schlicht, einen Perspektivenwechsel vorzunehmen, Repräsentationen des Zentrums dezidiert die Erfahrung und Stimme der Marginalisierten entgegenzusetzen, somit seine Dominanz in Frage zu stellen. Im Hinblick auf Literatur kann das geschehen, indem ein kanonisches Werk aus der Perspektive einer dort als marginalisiert und diskriminiert dargestellten Figur umgeschrieben wird. Bei dieser Grobskizzierung soll es jedoch bleiben; bei dem im folgenden vorgestellten Beispiel handelt es sich ebenfalls um ein writing back - eben zu Shakespeare -, das das, Wesen des Kolonialismus dramatisiert' (Zabus, Tempests 40): Aimé Césaires Une tempête/Ein Sturm (1969).

79 Nach einem ganz ähnlichen Prinzip der Stereotypisierung funktioniert übrigens Edward Saids Orientalism (1978). 


\section{Aimé Césaire, Une tempête/Ein Sturm (1969)}

Césaires Stück in drei Akten ist im Kontext eines ,schwarzen Theaters' verortet (,adaptation pour un théâtre nègre") und weist zwei grundlegende Änderungen bereits in der Liste der dramatis personae auf: Ariel ist nun ein mulattischer, Caliban ein schwarzer Sklave. Anders als bei Shakespeare wird Prosperos ,Kunst ${ }^{`}$ hier explizit mit kolonialem Expansionsstreben assoziiert (Akt I, Szene 2). Seine Bücher erschlossen ihm die Lage von Inseln, die er in Besitz zu nehmen gedachte, wobei ihm sein Bruder Antonio einen Strich durch die Rechnung machte und ihn zusammen mit Miranda auf der einer kleinen Insel aussetzte. Hier dient der von Prospero heraufbeschworene Sturm mehr oder minder der Ablenkung seiner Gegner von diesen Ländern: „Nous manigançâmes la tempête à laquelle tu viens d' assister qui préserve mes biens d'outre-mer et met en même temps ces sacripants en ma possession.“ (Césaire, Tempête 22) ${ }^{80}$ [,Wir haben den Sturm entfesselt, den du mit ansehen konntest, der meine transozeanischen Besitzungen schützt und diese Spitzbuben zugleich in meine Hand bringt." (Césaire, Sturm 13)] Schlagwörter wie „transozeanische[ ] Besitzungen“ und, vorher, „zukünftige[s] Reich“ (12) fallen hier auf den ersten Seiten und illustrieren den veränderten Kontext. Entsprechend tritt Shakespeares Haupthandlungsstrang um die Verschwörung von Antonio und Sebastian in den Hintergrund und ist bereits am Ende des zweiten Akts aufgedeckt und, aufgelöst'. Die Liebeshandlung um Miranda und Ferdinand folgt im Großen und Ganzen dem Shakespearschen Text; die Versöhnung zwischen Mailand und Neapel hat bei Césaire jedoch das Ziel, Prospero eine mächtige Allianz gegen den aufrührerischen Caliban zu verschaffen und ihn zu schützen. Aufgrund der neuen Kontextualisierung des Stücks kommt der Figurenkonstellation Prospero - Caliban - Ariel besondere Bedeutung zu.

Im ersten gemeinsamen Auftritt von Ariel und Prospero in Akt I, Szene 2, hat Ariel zwar ebenfalls sämtliche Befehle ausgeführt, ist von seiner Aufgabe jedoch angewidert. Anders als Shakespeares Luftgeist ist er in seinem Anliegen weniger vorsichtig: „Vous m'avez mille fois promis ma liberté et je l'attends encore.“ (Tempête 23) [,'Tausendmal habt Ihr mir die Freiheit versprochen, und immer noch warte ich darauf.“ (Sturm 14)] Auch nach seiner Zurechtweisung zeigt er keine übertriebene Dankbarkeit und Unterwürfigkeit, denn er ist sich der Abhängigkeit, in die ihn seine Rettung durch Prospero gebracht hat, bewusst. Prospero ist aber in Gedanken bereits mit Caliban beschäftigt, mit dem er ,ein Wörtchen zu reden hat', erscheint ihm sein schwarzer Sklave doch etwas zu ,emanzipiert'. Calibans erstes Wort unterstreicht diese Befürchtung, es ist der suahelische Begriff für Unabhängigkeit/Freiheit: „Uhuru“ (vgl. Zabus, „Calibanic Tempest“ 41). Suaheli ist die am weitesten verbreitete Verkehrssprache Ostafrikas; im Stück konnotiert sie eine ,einheimische ${ }^{6}$ schwarze Sprache; überdies assoziiert mit den z.T. bereits er-

80 Da weder die französische Originalausgabe noch die deutsche Übersetzung Zeilenangaben enthalten, gebe ich die jeweiligen Seitenzahlen an. 
folgreichen Unabhängigkeitsbestrebungen afrikanischer, aber auch karibischer Kolonien (vgl. Nixon 572). Ihre Verwendung durch Caliban ist als rebellische Geste gegen Prospero als Kolonisator und dessen Sprache beabsichtigt. Der nimmt prompt Anstoß an der „barbarischen Sprache“ (Sturm 15). Auch hier erfolgt ein Schlagabtausch von Beleidigungen, Drohungen und Flüchen, und auch hier verleiht Caliban seinem Anspruch als rechtmäßiger Inselbesitzer Ausdruck. Überdies bezeichnet er Prospero der Undankbarkeit, hat er ihn doch in die Geheimnisse der Insel eingeweiht. Inhaltlich deckt sich das soweit mit Shakespeares Caliban, allein der Ton ist ein völlig anderer, sarkastischer, mit dem er zu verstehen gibt, dass er Prospero durchschaut:

Gewiß doch! Anfangs hat der feine Herr mir schöngetan: mein lieber Caliban hier, mein guter Caliban dort! [...] Und jetzt kann ich dir gestohlen bleiben ... Caliban der Wilde! Caliban der Sklave! Bekanntes Rezept! Ist die Zitrone einmal ausgepreßt, wirft man die Schale weg. (Sturm 17) ${ }^{81}$

Noch deutlicher wird dies, als Caliban Prospero erklärt, er wolle nicht länger ,Caliban' genannt werden, weil das nicht sein Name sei, sondern Ausdruck von Hass und Unterdrückung. Seine Alternative:

Nenne mich X. Das ist besser. Als sagte man: Mensch ohne Namen. Noch genauer, der Mensch, den man seines Namens beraubt hat. Du sprachst eben von Geschichte. Nun, das ist Geschichte, und eine berüchtigte dazu. Jedesmal, wenn du mich rufst, wird mich das an die grundlegende Tatsache erinnern, daß du mir alles bis hin zu meiner Identität gestohlen hast. Uhuru!82 (Sturm 19)

Das ist nicht nur ein starker Abgang, sondern verortet diesen modernen Caliban zudem klar im Kontext schwarzer amerikanischer Bürgerrechtsbewegungen der 1960er Jahre; wir denken etwa an Malcolm X, bis 1964 Wortführer der radikalnationalistischen Organisation Black Muslims (respektive Nation of Islam) (vgl. Zabus, Tempests 47). Entsprechend begreift Prospero, dass er in Caliban einen Feind hat, gegen den er sich schützen sollte.

Zu Beginn von Akt II (Szene 1) findet eine Unterhaltung zwischen Ariel und Caliban statt, die die erwähnte historisch-politische Anspielung aufgreift und weiterführt. Hier kristallisieren sich zwei diametral entgegengesetzte Positionen heraus, als deren Vertreter die beiden Figuren fungieren, indem sie die Unterschiede zwischen gewaltlosen und radikalen Vertretern schwarzer Rechte reflektieren.

81 „C’est ça! Au début, Monsieur me cajolait: Mon cher Caliban par si, mon petit Caliban par là! [...] et maintenant je t'en fous ... Caliban la brute! Caliban l'esclave! Recette connue! l'orange pressée, on en rejette l'écorce!“" (Tempête 26)

82 „Appelle-moi X. Ça vaudra mieux. Comme qui dirait l'homme sans nom. Plus exactement, l'homme dont on a volé le nom. Tu parles d'histoire. Eh bien ça, c'est de l'histoire, et fameuse! Chaque fois que tu m'appeleras, ça me rappellera le fait fondamental, que tu m'as tout volé et jusqu'à mon identité! Uhuru!“ (Tempête 28) 
Beide haben dasselbe Ziel, Freiheit, nur, wie Ariel es formuliert, unterschiedliche Methoden, sie zu erlangen. Ariel betont die Gemeinsamkeiten - „mais après tout nous sommes frères dans la souffiance et l'esclavage, frères aussi dans l'espérance“ (Tempête 35) [,,doch schließlich sind wir Brüder, Brüder im Leiden und in der Sklaverei, Brüder auch in der Hoffnung“" (Sturm 23)] - und warnt Caliban vor Prosperos Rache, weil er es für seine Pflicht hält. Zugleich stellt er Calibans offen rebellische Haltung in Frage, glaubt er doch, gegen Prospero könne man nicht gewinnen. Caliban konfrontiert Ariel mit seinem Gehorsam und seiner Fügsamkeit, die ihm gerade nicht zu „Freedom now!“ (24) verhelfen wird. Während Caliban Gewalt propagiert, da er Prospero selbst für einen „Zerquetscher“ und „Zermalmer" (26) hält, und eher den Tod wählen würde als Demütigung und Ungerechtigkeit zu ertragen, zieht Ariel es vor, Prospero zu ändern, ihm die Unrechtmäßigkeit seines Tuns bewusst zu machen und ihn auf diese Weise gleichsam zu einem Gewissen zu ,erziehen'.

Was folgt, ist eine Anspielung auf Martin Luther Kings berühmte „I have a Dream"-Rede von 1963 in Washington D.C., in der er die bestehende Diskriminierung und Segregation der Schwarzen anprangerte, aber auch seinem Traum von und seiner Hoffnung auf ein friedliches, gleichberechtigtes Amerika Ausdruck verlieh. Zitiert sei lediglich ein Ausschnitt:

Ich habe einen Traum, dass eines Tages auf den roten Hügeln von Georgia die Söhne von früheren Sklaven und die Söhne früherer Sklavenhalter miteinander am Tisch der Bruderschaft sitzen können. Ich habe einen Traum, daß sich eines Tages selbst der Staat Mississippi, ein Staat, der in der Hitze der Ungerechtigkeit und Unterdrückung verschmachtet, zu einer Oase der Freiheit und Gerechtigkeit verwandelt.

Ich habe einen Traum, dass meine vier kleinen Kinder eines Tages in einer Nation leben werden, in der man sie nicht nach ihrer Hautfarbe, sondern nach ihrem Charakters beurteilen wird. ${ }^{83}$ (King 1999, 92)

Ariel formuliert seine Vision gewissermaßen im Kleinen, bezogen auf eine Gemeinschaft bestehend aus Caliban, ihm selbst, aber auch Prospero:

Wie oft hab ich davon geträumt, daß eines Tages Prospero, du und ich in brüderlicher Eintracht uns daran machten, eine neue wunderbare Welt aufzubauen, zu der ein jeder von uns seine ganz besonderen Fähigkeiten beitragen würde: Geduld, Vitalität und Liebe; Willenskraft und Strenge, von

83 „I have a dream that one day on the red hills of Georgia the sons of former slaves and the sons of former slave owners will be able to sit down together at the table of brotherhood. I have a dream that one day even the state of Mississippi, a state sweltering with the heat of injustice $[\ldots]$, sweltering with the heat of oppression, will be transformed into an oasis of freedom and justice. I have a dream [...] that my four little children will one day live in a nation where they will not be judged by the color of their skin but by the content of their character." (King 2006, $558-59)$ 
jenem leichten Traumgewölk zu schweigen, ohne das die Menschheit an Erstickung zugrunde ginge. ${ }^{84}$ (Sturm 25)

Der Wunsch nach Gleichberechtigung und Integration, den Martin Luther King besonders eindringlich artikulierte, ist hier in der Gemeinsamkeit von ehemaligem Unterdrücker und ehemaligen Unterdrückten impliziert.

Es ist jedoch Caliban, der sich mit seinen Methoden durchsetzt. Im dritten und letzten Akt trifft er auf Stephano und Trinculo (Szene 2) - zur Erinnerung: bei Shakespeare geschah das bereits im zweiten Akt -, die ihn hier tatsächlich für einen ,authentischen Indianer aus der Karibik ${ }^{6}$ (Tempête 59-60) halten und sofort daran denken, ihn zu zivilisieren bzw. ihre „zivilisatorische Mission“ - in Anlehnung an Rudyard Kipling könnte man sagen, die ,Bürde des weißen Mannes` - zu erfüllen (Sturm 42). Auch hier schließt sich Caliban ihnen zunächst an, um mit ihrer Hilfe Prospero loszuwerden. Anders als Shakespeares Figur erkennt er jedoch sehr viel schneller, wie es um die Intelligenz seiner vermeintlichen Verbündeten bestellt ist und kommt zu dem Schluss, dass er den Gegner allein bekämpfen muss. Als der sich ihm allerdings mit entblößter Brust entgegenstellt, tötet Caliban ihn nicht; will er doch nicht zum Mörder werden an einem Feind, der sich nicht verteidigen kann. Dieses Zögern nutzt Prospero, um Caliban von Ariel gefangennehmen zu lassen. Damit ist die finale Konfrontation eingeleitet, denn die restlichen Seiten des Textes werden von Caliban und Prospero bestritten. Caliban lehnt ein Friedensangebot seines Gegners ab und wirft ihm in einer längeren Rede seine Unterdrückungspraktiken vor, die auf eine Internalisierung negativer Stereotype abzielen, das heißt: dem kolonialisierten Subjekt ein negatives Selbstbild einimpfen (wie von Frantz Fanon beschrieben). ${ }^{85}$ Lassen wir Caliban selbst zu Wort kommen (Akt III, Szene 5):

Und du hast mich derart belogen, / belogen über die Welt, belogen über mich selbst, / daß du mir schließlich / ein Bild meiner selbst aufgezwungen hast: / Das Bild eines Unterentwickelten, wie du sagst, / eines Minderbemittelten, / so mich zu sehen, hast du mich gezwungen, / und dieses Bild, ich hasse es! Und es ist falsch!86 (Sturm 62-63)

84 "J'ai souvent fait le rêve exaltement, qu'un jour, Prospero, toi et moi, nous entreprendrions, frères associés, de bâtir un monde merveilleux, chacun apportant en contribution ses qualités propres: patience, vitalité, amour, volonté aussi, et rigeur. Sans compter les quelques bouffées de rêve sans quoi l'humanité périrait d'aphyxie." (Tempête 38)

85 Denken wir zurück an Shakespeares Caliban, so ist es durchaus möglich, eine solche Internalisierung auch bei ihm zu konstatieren, die sich darin zeigt, dass er zwar von Prosperos Herrschaft befreit sein möchte, sich zu diesem Zwecke aber den Nächstbesten freiwillig unterwirft, seine Vorstellung von Freiheit also korrumpiert wurde (vgl. 2.3).

86 „Et tu m'as tellement menti, / menti sur le monde, menti sur moi-même, / que tu a fini par m’imposer / une image de moi-même: / Un sous-développé, comme tu dis, / un sous-capable, / voilà comment tu m'as obligé à me voir, / et cette image, je le hais! Et elle est fausse!“" (Tempête 88) 
Das Infragestellen bzw. aggressive Angreifen der Definitionsmacht des Herrschers stellt einen besonders deutlichen Akt des Widerstands dar, der zudem die Notwendigkeit eines Umschreibens der als ,falsch“ enttarnten Kolonialgeschichte betont. Konfrontiert mit Calibans Hass und seinen Drohungen, Prospero zu „fassen [zu] kriege[n]“ (62), letztlich mit seinem eigenen Scheitern, ändert dieser sein „Ich bedaure dich!““ (63) kurz darauf ab in ein „Nun denn, ich auch, ich hasse dich / Denn du hast mich / zum ersten Male / an mir selber zweifeln lassen.“"87 (64) Er hält es jedoch für seine Pflicht, auf der Insel zu bleiben, um sie lebensfähig zu halten (hier kommt offenbar wieder die ,Bürde des weißen Mannes“ zum Tragen), aber auch, um seine Herrschaft weiterhin ausspielen zu können. So bleibt er mit Caliban allein zurück und schwört, er werde fortan Gewalt mit Gewalt vergelten dies deutet bereits an, dass seine Machtposition erheblich geschwächt ist. In einer Art Epilog sehen wir Prospero dann, Jahre später, alt, abgekämpft mit fahrigen Gesten und tonloser Stimme. Die Natur scheint sich gegen ihn verschworen zu haben, er fühlt sich von ihr bedroht (laut Caliban verkörpert er die ,Anti-Natur'), sogar das Klima ist kälter geworden, und er muss sein Feuer nun selbst schüren. Trotzig hält er an seiner Mission fest: „Aber ich werde mich verteidigen ... Ich lasse mein Werk nicht zugrunde gehen ...! Ich werde die Zivilisation verteidigen!“88 (Sturm 66) Doch in Anbetracht der Umstände ist klar, dass seine Macht (und seine Herrschaft über die Insel) längst gebrochen ist und er zunehmend senil und/oder wahnsinnig wird. Ein Sturm schließt mit Calibans: „DIE FREIHEIT OHÉ! DIE FREIHEIT!' ${ }^{\text {(89 }}$ (Sturm 66), das einmal mehr unterstreicht, wie sich das Verhältnis zwischen Kolonisator und Kolonisiertem gewandelt hat und letztlich Calibans Sieg über einen Prospero, der am Ende seiner Privilegien enthoben ist, suggeriert. Sein Aufstand verursacht den eigentlichen, titelgebenden Sturm im Stück und macht Caliban zum erfolgreichen, schwarzen Revolutionär (vgl. Zabus, Tempests 54). ${ }^{90}$ Somit ist Césaires Drama - wie auch sein Protagonist - im politischen Kontext der Zeit klar verortet.

Im Zuge einer zunehmenden Aufweichung oder gar Überwindung postkolonialer Dichotomien mag das unmittelbar politisch motivierte Interesse an den kolonialen Implikationen von Shakespeares Sturm etwas abgeflaut sein (vgl. Nixon 577), doch findet eine imaginative Auseinandersetzung mit und Hinterfragung von diesem klassischen Werk der englischen Literatur mit veränderten - z.B. postmodernen oder feministischen - Schwerpunkten nach wie vor statt und zeugt von

87 „Eh bien moi-aussi je te hais! / Car tu es celui par qui pour / la première fois j’ai douté de / moi-même.“ (Tempête 90)

88 „Mais je me défendrai ... je ne laisserai pas périr mon œuvre...Je défendrai la civilisation!“ (Tempête 92)

89 „LA LIBERTÉ OHÉ, LA LIBERTÉ!““ (Tempête 92)

90 „Caliban is at once Césaire's mouthpiece, the embodiment of the concept of Négritude as well as the Afro-Caribbean and African American colonized subject. He also documents all to himself the shift from Caliban as native Indian/Caribbean/cannibal to Black African/colonised slave on to the triumphant Third World revolutionary of the late sixites." (Zabus, Tempests 54) 
der Nachhaltigkeit eines ,alten' Stücks in unserer ,neuen' Welt des 20. und 21. Jahrhunderts.

\section{Verwendete Literatur}

\section{Primärwerke}

Césaire, Aimé. Une tempête. Adaptation de ,La Tempête' de Shakespeare pour un théatre nègre. Paris: Éditions du Seuil, 1969.

---. Ein Sturm. Stück für ein schwarzes Theater. Übersetzung von Monika Kind. Berlin: Wagenbach, 1970.

---. A Tempest. Based on Shakespeare's The Tempest. Adaptation for a Black. Theatre. Translated from the French by Richard Miller. New York: TCG Translations, 2002.

Shakespeare, William. The Tempest. Hg. Frank Kermode [1954]. Repr. 1990. London: The Arden Shakespeare, 1964.

---. The Tempest. Hg. Alden T. Vaughan und Virginia Mason Vaughan. Third Series. Repr. 2007. London: The Arden Shakespeare, 1999.

---. Der Sturm. Englisch und Deutsch. Hg. Levin L. Schücking. Übersetzung von August Wilhelm Schlegel und Ludwig Tieck. O.O. [Reinbek]: Rowohlt, 1962.

Sekundärwerke

Ashcroft, Bill, Gareth Griffiths, Helen Tiffin. The Empire Writes Back: Theory and Practice in Postcolonial Literatures. London; New York: Routledge, 1989.

Greenblatt, Stephen. „Culture.“ Hg. Frank Lentricchia und Thomas McLaughlin. Critical Terms for Literary Study. $2^{\text {nd }}$ ed. Chicago; London: University of Chicago Press, 1995. 225-232.

Kastan, David Scott. ,„The Duke of Milan / And his Brave Son': Old Histories and New in The Tempest." Thorne 226-244.

Kelley, Robin D.G. „Poetry and the Political Imagination: Aimé Césaire, Negritude and the Applications of Surrealism. " Césaire, A Tempest vii-xvi.

King, Martin Luther.

Ich habe einen Traum. Hg. Hans-Eckehard Bahr und Heinrich Grosse. Zürich; Düsseldorf: Benziger, 1999.

---. „Address to the March on Washington. August 28, 1963.“ Widmer 556-560.

Kullmann, Thomas. William Shakespeare: Eine Einfübrung. Berlin: Schmidt, 2005.

Lamming, George. „A Monster, A Child, A Slave.“ George Lamming. The Pleasures of Exile. London: Allison \& Busby, 1960. 95-117. 
McLeod, John. Beginning Postcolonialism. Manchester: MUP, 2000.

Nixon, Rob. „Caribbean and African Appropriations of The Tempest.“ Critical Inquiry 13 (Spring 1987): 557-578.

Pape, Walter und Frederick Burwick, Hg. The Boydell Shakespeare Gallery. Bottrop: Pomp, 1996.

Schabert, Ina, Hg. Shakespeare-Handbuch. 3. Aufl. Stuttgart: Kröner, 1992.

Scott, Grant F. „To Play the King: Illustrations from The Tempest in the Boydell Shakespeare Gallery." Pape und Burwick 113-122.

Singh, Jyotsna G. „Caliban versus Miranda: Race and Gender Conflicts in Postcolonial Rewritings of The Tempest. "Thorne 205-225.

Thorne, Alison, Hg. Shakespeare's Romances: New Casebooks. Basingstoke: Palgrave Macmillan, 2003.

Vaughan, Alden T. und Virginia MasonVaughan. Shakespeare's Caliban: A Cultural History. Cambridge: CUP, 1991.

wa Thiong’o, Ngugi. „The Language of African Literature.“ Williams und Chrisman 433-455.

Widmer, Ted, Hg. American Speeches: Political Oratory from Abraham Lincoln to Bill Clinton. New York: The Library of America, 2006.

Williams, Patrick und Laura Chrisman, Hg. Colonial Discourse and Post-Colonial Theory: A Reader. New York [u.a.]: Harvester Wheatsheaf, 1993.

Zabus, Chantal. „A Calibanic Tempest in Anglophone and Francophone New World Writing." Canadian Literature 104 (1985): 35-50.

Zabus, Chantal. Tempests after Shakespeare. New York: Palgrave, 2002. 